Hadi Asgharzadeh Shirazi, Majidreza Ayatollahi, Alireza Karimi* and Mahdi Navidbakhsh

\title{
A comparative finite element analysis of two types of axial and radial functionally graded dental implants with titanium one around implant-bone interface
}

DOI 10.1515/secm-2015-0392

Received September 16, 2015; accepted November 18, 2015; previously published online April 6, 2016

\begin{abstract}
Functionally graded biomaterials (FGBMs) have received significant attention in the recent years as potential candidates for the next generation of dental implant improvement. This happened due to their unique advantages and their ability to satisfy the requirements of both biomechanical and biocompatibility properties simultaneously. This study was aimed to analyze the effects of two radial and axial FGBM dental implants on the stress distribution near the dental implant-bone interface under a static load using finite element method (FEM). The model was restrained on a base supporting bone and vertically loaded with a force of $100 \mathrm{~N}$ on the top of the abutment. In the FGBM models, the implants are made of a combination of bioceramic and biometal composition, with properties that change gradually and continuously in the radial and axial directions. The numerical results indicated that the use of both radial and axial FGBM dental implants reduced the
\end{abstract}

\footnotetext{
*Corresponding author: Alireza Karimi, Department of Mechanical Engineering, Kyushu University, 744 Motooka, Nishi-ku, Fukuoka 819-0395, Japan, e-mail: karimia@outlook.com; and Tissue Engineering and Biological Systems Laboratory, Department of Biomechanics, School of Mechanical Engineering, Iran University of Science and Technology, Tehran 16887, Iran

Hadi Asgharzadeh Shirazi: Fatigue and Fracture Research Laboratory, Center of Excellence in Experimental Solid Mechanics and Dynamics, School of Mechanical Engineering, Iran University of Science and Technology, Tehran, Iran; and Tissue Engineering and Biological Systems Laboratory, Department of Biomechanics, School of Mechanical Engineering, Iran University of Science and Technology, Tehran 16887, Iran

Majidreza Ayatollahi: Fatigue and Fracture Research Laboratory, Center of Excellence in Experimental Solid Mechanics and Dynamics, School of Mechanical Engineering, Iran University of Science and Technology, Tehran, Iran

Mahdi Navidbakhsh: Tissue Engineering and Biological Systems Laboratory, Department of Biomechanics, School of Mechanical Engineering, Iran University of Science and Technology, Tehran 16887, Iran
}

maximum von Mises stress in the cortical and the cancellous bones in comparison with the common titanium one, which leads to faster bone regeneration and early stabilization of dental implant system. The findings of the present study may have implications not only for understanding the stresses and deformations around the implant-bone interface but also for improving the performance as well as application of FGBMs in dental implant materials.

Keywords: dental implant; finite element analysis; functionally graded biomaterial; implant-bone interface; von Mises stress.

\section{Introduction}

The purpose of modern dentistry is to restore the patient to normal function, health, and aesthetics. Dental implants can be a bold restoration option for people in a suitable general oral health who have lost a tooth because of periodontal disease, an injury, or some other reasons. Due to the lack of periodontal ligament transfer in dental implant, the forces of chewing will transfer directly to the surrounding bones near the implant. Therefore, much effort has been devoted to investigate the relation between the characteristics of dental implant and the stress distribution near the dental implant-bone interface [1-5]. The most common cause of failure in dental implant is inadequate bone formation around the biomaterial immediately after implantation [6]. Therefore, the development of new biomaterials for dental implants is one of the most challenging tasks for materials science today. A single composition with a uniform structure cannot satisfy the requirements for some biomedical applications, as dental biomaterials must meet several important criteria and have appropriate properties, such as biocompatibility, with a known degradation rate, osteoconductivity, strength, corrosion resistance, elastic modulus, fatigue durability, and close chemical similarity to that of the biological apatite present in human hard tissues [7-10]. Functionally graded 
biomaterials (FGBMs) have recently been introduced in dental implants owing to their unique advantages and ability to satisfy the requirements of both biomechanical and biocompatibility properties simultaneously. FGBM is a nonhomogeneous biomaterial composed of bioceramic and biometal; its properties such as elasticity modulus, mass density, and thermal conductivity vary smoothly and continuously through the biomaterial. Due to the high applicability of FGBM composite biomaterials, a number of researches have been dedicated many times to the biomedical applications of FGBM composites [11-16]. In the case of dental implants, FGBM is usually considered to comprise a mixture of titanium and bioactive hydroxyapatite (HA) due to its biocompatibility, bioactivity, and structural resemblance to that of the organic part of the teeth and body bones. HA, which is found in the teeth and bones of animals as well as humans, is an osteophilic material that not only does not absorb but also does not solve in the body solutions. Furthermore, it stimulates natural bone production, such that bony structures fill the spaces around the implant and make a good bond with bones in a direct connection. As this material has a good biocompatibility with the tissues forming the human body, it is widely used as a coating material for dental implants and prostheses. HA can be produced by two methods: organic synthesis [17-20] and extraction from a natural source [2123]. The physical and mechanical properties of HA vary according to different conditions and synthesis methods. Many studies have been performed to improve the properties of HA, such as assessing the effects of sintering and annealing processes on HA properties [23-26]. Dental implants with functionally graded structures are composed of titanium and various bioceramics, including HA, which has been fabricated to satisfy the requirements of both mechanical and biocompatibility in previous investigations [27-29]. However, a small number of studies have been conducted to analyze FGBM dental implants using finite element method (FEM) [30-33], which obviously is considered as a simple and inexpensive way for finding the stress distribution.

It has been reported that the mechanical properties of FGBM implant are varied axially with a single power-law function. The purpose of this paper is to investigate the effects of both radial and axial FGBM dental implants on the stress distribution near the dental implant-bone interface based on one and two power-law functions, respectively. To do this, the results obtained from FGBM dental implants in the radial and axial directions were compared to those of common titanium implants. As the mandible bone is more vulnerable to damage than the implant, this study has concentrated on the bone stress distribution.

\section{Materials and methods}

The geometry of a commercial solid screw implant (Nobel Biocare $^{\circledR}$, Branemark, Gothenburg, Sweden) was used as a reference to model a cylindrical implant and a solid abutment, which were developed to one unit. The modeling of a human mandible, including the cancellous core surrounded by the cortical layer with the width of $1.5-2 \mathrm{~mm}$, was simulated based on the CT scan inputs. The ABAQUS 6.10 software (SIMULIA Corporation, Providence, RI, United States) was utilized to simulate and obtain the bone stress distribution. In order to define the contact between the implant and bone, it was assumed that the osseointegration existed completely and the simulation contact was considered to be perfectly bonded. A concentrated force of $100 \mathrm{~N}$ was vertically applied on the center of abutment's top surface, and the model was restrained on a base supporting bone. The details of the simulation model are illustrated in Figure 1.

In the radial FGBM implant, the mechanical properties changed gradually and continuously in the radial direction according to a special function. In other words, the center of Ti/HA radial FGBM implant was considered to be a titanium biometal, and its properties changed based on a predefined function up to the outer layer (in contact with the bone), which is an HA bioceramic and a bone-compatible phase. In this study, Poisson's ratio $v$ is considered constant and the elasticity modulus $E$ of the implant is assumed to vary radially based on a continuously parabolic (PFGM) nonlinear equation [34]:

$$
\left\{\begin{array}{l}
E(r)=E_{0}\left(1-n R^{\beta}\right) \\
n=1-\frac{E_{\text {out }}}{E_{0}}, \quad R=\frac{r}{b}
\end{array}\right.
$$

where $E_{0}$ is the reference value of $E$ related to elasticity modulus of titanium biometal, $r$ is the radial coordinate, $b$ is the outer radius, and $n$ and $\beta$ are the "two" material parameters. In addition, $E_{\text {out }}$ indicates the elasticity modulus of the outer layer related to the elasticity modulus of bioceramics. Figure 2A shows the nondimensional elasticity modulus $\left(E / E_{0}\right)$ along the radial direction for different values of $n$ and $\beta$. According to this figure, for a fixed value of $R(0<R<1)$ and $n=0.4$, the nondimensional elasticity modulus decreases by reducing $\beta$, while for $n=-0.4$, it would be vice versa.

In the axial FGBM implant, the material profile changes continuously along the longitudinal direction from Ti biometal-rich in one end to HA bioceramic-rich in the other end, which is implanted inside the mandible bone to achieve the optimal mechanical properties and 


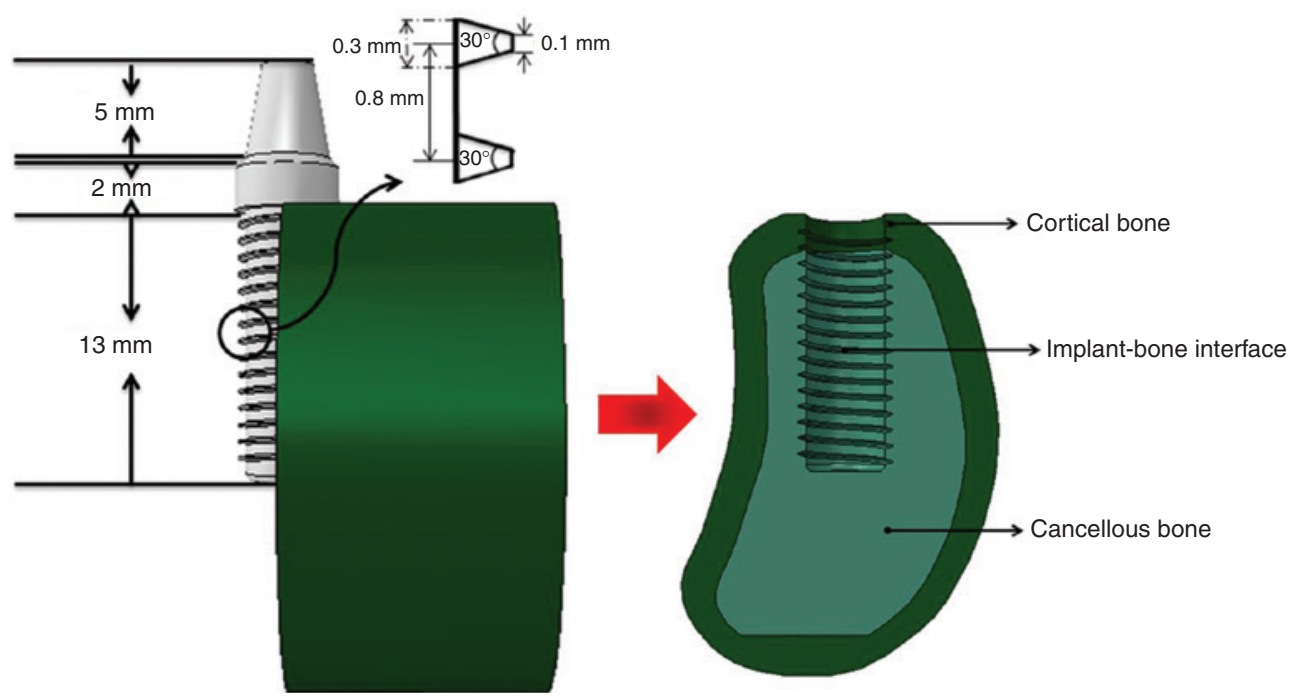

Figure 1: Details of the dental implant/bone system simulation.


Figure 2: Variations in material properties for $(A)$ the radial and (B) the axial FGBM configurations.

biocompatibility. Previous studies have concentrated on the FGBM with a single power-law function only along the longitudinal direction [30-33]. In this study, volume fraction should be defined using two power-law functions in order to reach the aim of smooth bone stress distribution among all the interfaces. The two power-law or sigmoid functions (SFGM) are defined by [35]:

$$
\left\{\begin{array}{l}
g_{1}(z)=1-\frac{1}{2}\left(\frac{\frac{h}{2}-z}{\frac{h}{2}}\right)^{\eta} \quad \text { for } 0 \leq z \leq \frac{h}{2} \\
g_{2}(z)=\frac{1}{2}\left(\frac{\frac{h}{2}+z}{\frac{h}{2}}\right)^{\eta} \quad \text { for }-\frac{h}{2} \leq z \leq 0
\end{array} .\right.
$$

The elasticity modulus can be calculated also by:

$$
\left\{\begin{array}{ll}
E(z)=g_{1}(z) E_{1}+\left[1-g_{1}(z)\right] E_{2} & \text { for } 0 \leq z \leq \frac{h}{2} \\
E(z)=g_{2}(z) E_{1}+\left[1-g_{2}(\mathrm{z})\right] E_{2} & \text { for }-\frac{h}{2} \leq z \leq 0
\end{array},\right.
$$

where $E_{1}$ and $E_{2}$ are the elasticity moduli of the top end $(z=h / 2)$ related to the titanium biometal and bottom end $(z=-h / 2)$ related to HA bioceramic of the axial FGBM implant, respectively. For different values of $\eta$, the elasticity modulus along the longitudinal direction is plotted in Figure 2B. According to this figure, in the range from 0 to 0.5 of nondimensional length $(z / h)$, the elasticity modulus decreases by reducing $\eta$, while in the range between -0.5 and 0 of nondimensional length $(z / h)$, the reverse holds true. For the FE modeling of radial and axial FGBM implants, the variations of material properties were implemented by 20 layers in the radial and axial directions while the material properties for each layer were constant. 
All the materials in this study have been assumed to be isotropic and homogenous; although it has been indicated that biological structures, such as the bone, are not isotropic, this is a common assumption in numerical modeling of oral structures [36, 37]. Table 1 represents the mechanical properties of materials used in this work.

In order to achieve reliable data, the convergence of $\mathrm{FE}$ outcomes was evaluated. For the convergence test and the modification of mesh size, the maximum von Mises stress was obtained in the case of titanium implant. The calculation shall be reported for finer mesh sizes until the relative errors between two successive analyses become $<1 \%$. Details of element and node numbers for the finest mesh model are listed in Table 2. Moreover, as an additional verification, a comparison between the results of this work and that of Kong et al. [38] was made under the same simulation conditions. In fact, in their study, models were constrained in all directions at the nodes on the mesial and distal bone and the static force of 100 was applied axially to the middle point in the center of the superstructure. Table 3 indicates a comparison between the values of maximum von Mises stress in the present study and the ones given by Kong et al. [38], in which a good agreement was seen between the results.

\section{Results}

In the case of radial FGBM implant, the stress distribution of bone was evaluated in response to the radial FGBM implant, in which the mechanical property, i.e. modulus of elasticity, of dental implant varies gradually in the radial direction. The maximum von Mises stress versus

Table 1: Material properties in the simulation models.

\begin{tabular}{lrrr}
\hline Materials & $\begin{array}{r}\text { Modulus of } \\
\text { elasticity (GPa) }\end{array}$ & $\begin{array}{r}\text { Poisson's } \\
\text { ratio }\end{array}$ & References \\
\hline Titanium & 110 & 0.35 & {$[38-44]$} \\
Cortical bone & 14 & 0.30 & {$[38-44]$} \\
Cancellous bone & 1.37 & 0.31 & {$[38-44]$} \\
Hydroxyapatite & 40 & 0.27 & {$[30]$} \\
\hline
\end{tabular}

Table 2: Number of elements and nodes in dental implant models.

\begin{tabular}{lrr}
\hline Sample & Number of nodes & Number of elements $^{\text {a }}$ \\
\hline Radial FGBM & 23,458 & 116,751 \\
Axial FGBM & 22,454 & 108,283 \\
\hline
\end{tabular}

a Linear tetrahedral of type C3D4.
Table 3: A comparison between the results in present study and those reported in Ref. [38] under the same simulation.

\begin{tabular}{lrrr}
\hline Region & \multicolumn{2}{c}{ Maximum von Mises stress (MPa) } & \multirow{2}{*}{ \% Error } \\
\cline { 2 - 2 } & Present study & Kong et al. [38] study & \\
\hline Cortical bone & 4.107 & 4.097 & 0.244 \\
Cancellous bone & 1.430 & 1.431 & 0.070 \\
\hline
\end{tabular}

the volume fraction index $n$ in the cortical and cancellous bones is presented in Figure 3. As can be observed from this figure, in the case of radial FGBM implant, the maximum von Mises stress decreases for $n<0$ and increases for $n>0$ compared to the titanium one $(n=0)$ in both the cortical and cancellous bones. However, the maximum von Mises stress continuously decreases in a range of $n=-0.4$ to $n=0.4$ by increasing $n$.

In the case of axial FGBM implant, the stress distribution of bone was investigated in response to the axial FGBM implant, in which the mechanical property, i.e. modulus of elasticity, of dental implant varies gradually and continuously along the longitudinal direction. Figure 4 illustrates the variations of maximum von Mises stress versus the volume fraction index $\eta$ in the axial FGBM implant. According to this figure, the maximum von Mises stress in the axial FGBM implant system decreases for all values of $\eta$ in comparison with common Ti one. Moreover, this figure can be divided into two intervals; in the first interval of $\eta(1-5)$, the maximum von Mises stress changes rapidly compared to its variations in the second interval (5-10). This behavior is related to the mechanical properties, such as elastic modulus, of axial FGBM implant, which were described in equation 2 and Figure 2B. As shown in Figure 2B, the curves of the elastic modulus variations of axial FGBM implants

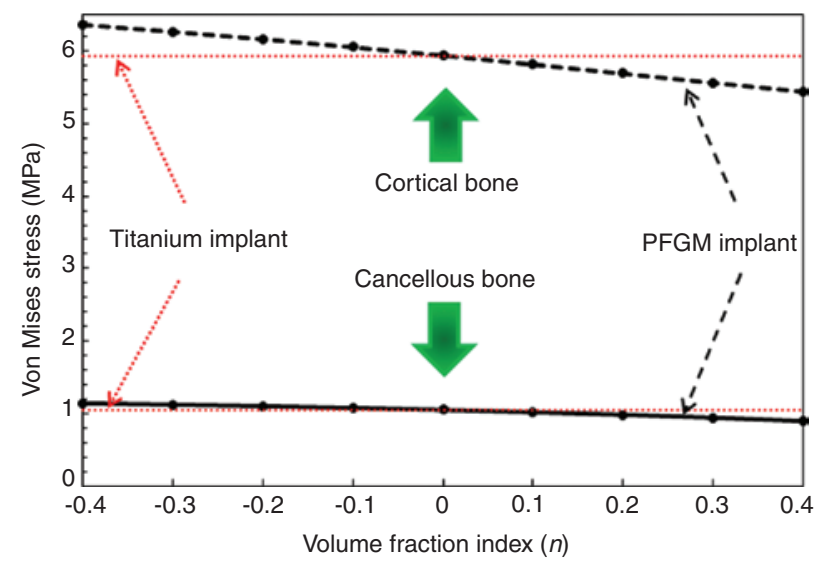

Figure 3: Maximum von Mises stress of cortical and cancellous bones in response the radial FGBM implant $(\beta=0.9)$. 


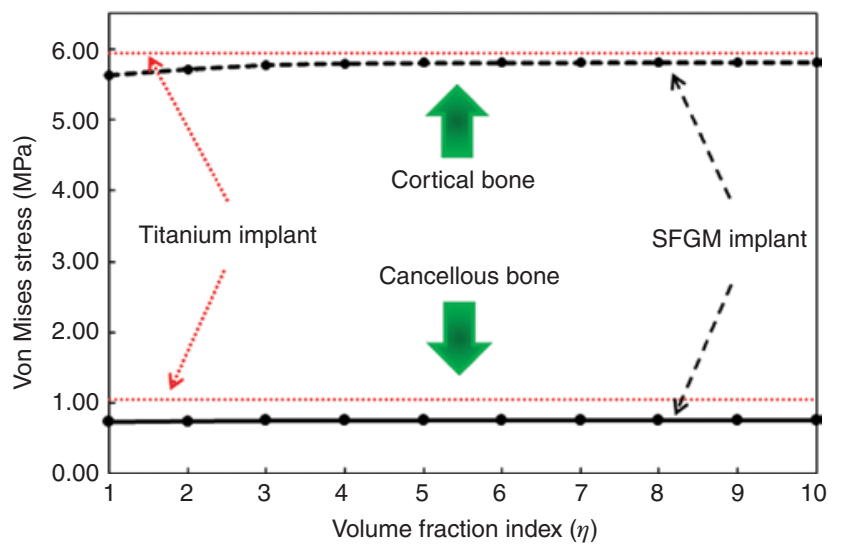

Figure 4: Maximum von Mises stress of cortical and cancellous bones in response to the axial FGBM implant.

vary closely to each other in the range of $\eta$ between 5 and 10. Therefore, it is expected that the curves of maximum von Mises stress versus the volume fraction index also vary closely to each other in this range of $\eta$, i.e. $5 \leq \eta \leq 10$. Figure 4 exhibits a good coincidence with this expectation. The summary of Figures 3 and 4 is listed in Table 4.

Overall, for the case study, Figure 5 displays the maximum von Mises stress of mandible bone in response to the radial FGBM implants (where the outer layer is HA, i.e. $n=0.64$ ) and the axial one (where $\eta=1$ ). Based on this figure, the maximum von Mises stress values of radial and axial Ti/HA FGBMs, as a bone-compatible phase, have a vivid reduction as compared to the titanium one in the cortical and cancellous bones, and this reduction leads to a faster bone regeneration and early stabilization of the implant, based on the previous reports [39].

On the other hand, the study on bone stress distribution can play a crucial role in the assessment of dental implant system performance in clinical applications due to the vulnerability of the bone. Figure 6 shows the stress distribution in the cortical and cancellous bones for the Ti/HA FGBM implants and for the titanium one. According to this figure, the maximum value of bone stress takes place in the titanium implant compared to FGBM ones. In other words, the maximum von Mises stress of bone is reduced when the FGBM implant is used; however, the

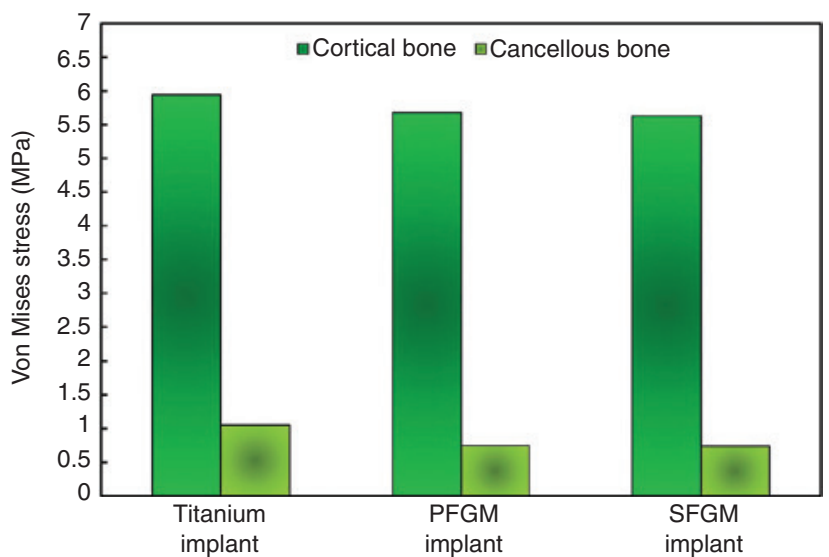

Figure 5: A comparison between both the radial $\left(E_{\text {out }}=40\right)$ and the axial $(\eta=1)$ FGBM implants and titanium one in terms of maximum von Mises stress in cortical and cancellous bones.

intensity of stress distribution of jaw bone increases in the radial and axial FGBM implants.

Figure 7 illustrates the position of maximum von Mises stress in response to the radial and axial FGBM and titanium implants in the cancellous bone. As shown in this figure, the maximum von Mises stress occurs in the last threads and bottom of implant-cancellous bone interface for both the radial FGBM and titanium implants. In contrast, the maximum von Mises stress was observed in the first threads and top of implant-cancellous bone interface for the axial FGBM implant. In the axial FGBM implant, according to Equation 2 and Figure 2B, as the mechanical properties, such as elastic modulus, increase from the bottom end to the top one, it was predictable that the maximum stress occurs in the superior region of the cancellous bone. Figure 7 indicates a good consistency compared to the reports from previous investigations [3033, 38-44].

\section{Discussion}

The results of the present study were used for the assessment of biomechanical compatibility of the FGBM dental

Table 4: Maximum von Mises stress (MPa) of cortical and cancellous bones in response to the titanium (Ti), radial and axial FGBM implants with various $n$ and $\eta$ values, respectively.

\begin{tabular}{|c|c|c|c|c|c|c|c|c|c|c|}
\hline Radial FGBM parameters & $n=-0.4$ & $n=-0.3$ & $n=-0.2$ & $n=-0.1$ & $n=0$ & $\mathrm{Ti}$ & $n=0.1$ & $n=0.2$ & $n=0.3$ & $n=0.4$ \\
\hline Cortical bone & 6.370 & 6.272 & 6.170 & 6.059 & 5.943 & 5.943 & 5.821 & 5.695 & 5.567 & 5.444 \\
\hline Cancellous bone & 1.144 & 1.124 & 1.103 & 1.078 & 1.050 & 1.050 & 1.019 & 0.984 & 0.944 & 0.896 \\
\hline Axial FGBM parameters & $\eta=1$ & $\eta=2$ & $\eta=3$ & $\eta=4$ & $\eta=5$ & $\eta=6$ & $\eta=7$ & $\eta=8$ & $\eta=9$ & $\eta=10$ \\
\hline Cortical bone & 5.634 & 5.720 & 5.780 & 5.804 & 5.810 & 5.813 & 5.815 & 5.817 & 5.819 & 5.820 \\
\hline Cancellous bone & 0.7305 & 0.7419 & 0.7501 & 0.7533 & 0.7549 & 0.7558 & 0.7563 & 0.7568 & 0.7571 & 0.7574 \\
\hline
\end{tabular}




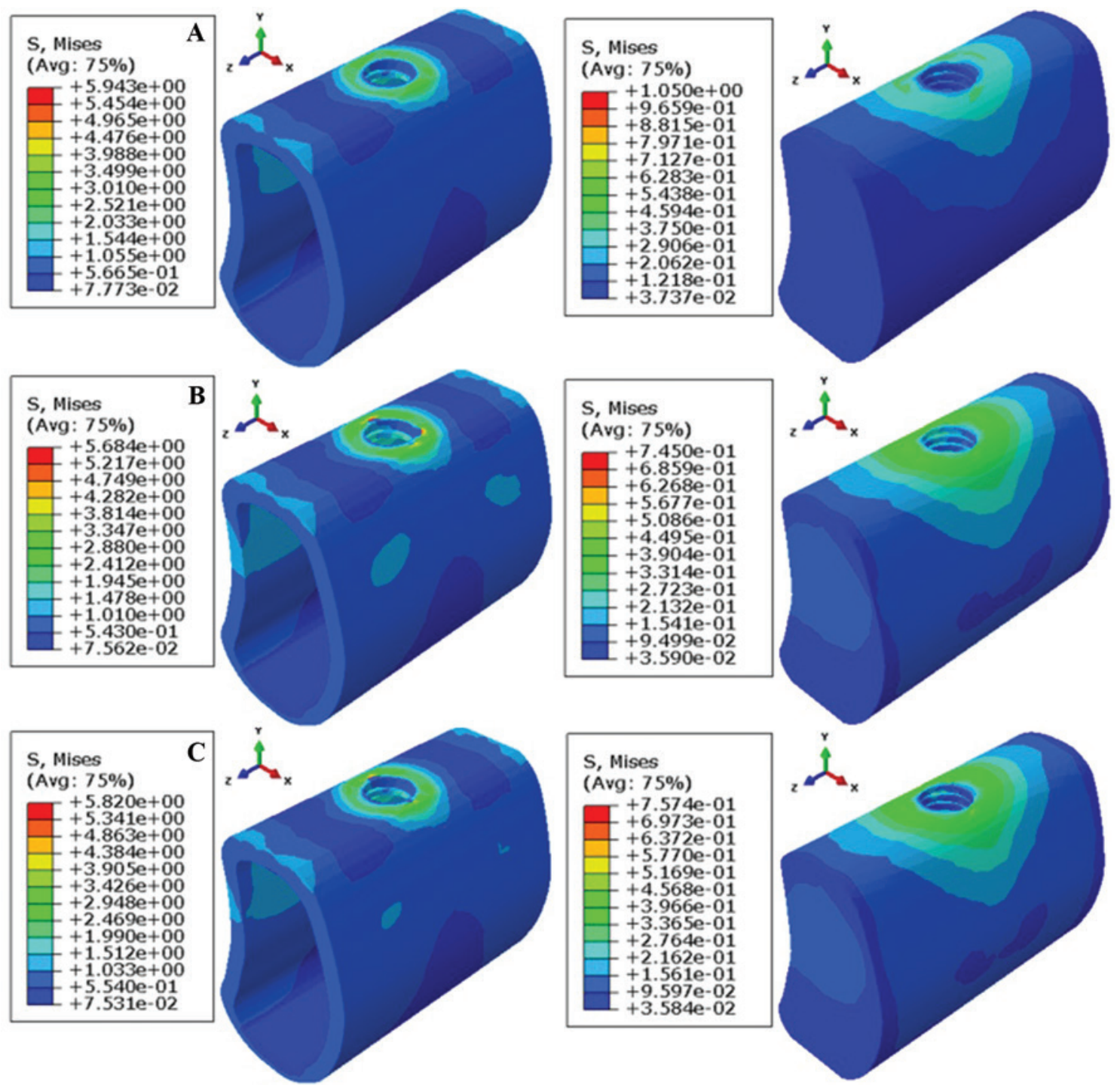

Figure 6: The von Mises stress distribution of jaw bone in (A) titanium, (B) PFGM ( $E_{\text {out }}=40 \mathrm{GPa}$ ), and (C) SFGM ( $\left.\eta=10\right)$ implants.

implant. In most of the previous studies on FGBM dental implant [30-33], the mechanical property, i.e. elastic modulus, of FGBM implant is assumed to vary axially based on a single power-law function, but in this study, these properties are considered to change radially and axially according to single and two power-law functions, respectively. In all assessments, the maximum von Mises stress of the mandible bone appears near the neck of dental implant, which has a good agreement with reports of the previous literature [30-33, 38-44]. In the radial FGBM case, a significant reduction in the maximum von Mises stress of the cancellous bone is observed in a way that this reduction leads to higher stability and faster bone regeneration, and as a result, the performance of dental implant system improves. In addition, due to the presence of a biocompatible and bioactive bioceramic, such as HA, as bone-compatible phase, in the outer layer and in direct contact with the bone, the stimulation and bone growth increase near the dental implant-bone interface and hence the healing time is reduced. In the axial FGBM case, a significant reduction in the maximum von Mises stress of the cancellous bone is also seen. The mechanical properties vary in the longitudinal direction gradually and continuously from Ti biometal-rich in one end to HA bioceramic-rich in the other end. This causes the implant to have a higher strength at top regions and first threads of dental implant (where stress is high) and the biocompatibility and bioactivity properties to increase at the bottom areas (where the stress is low). Moreover, the FGBM implant can significantly reduce the risks of failure modes, such as decomposition of HA, amorphous calcium phosphate (ACP) formation, weak adhesion 

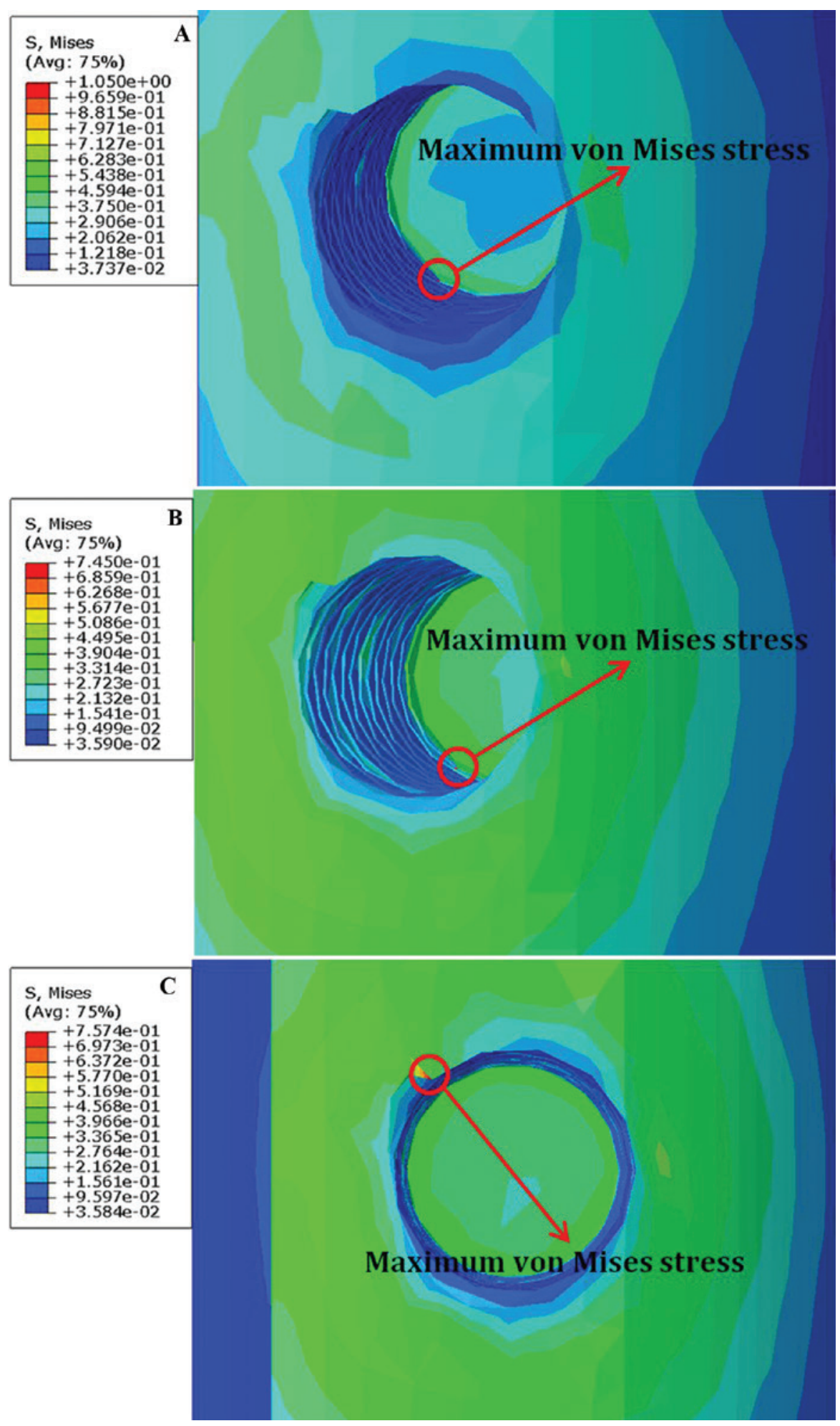

Figure 7: The position of maximum von Mises stress in cancellous bone for cases of (A) titanium, (B) PFGM ( $\left.E_{\text {out }}=40\right)$, and (C) SFGM $(\eta=10)$ implants.

strength between the HA coating and the titanium substrate, and cracking and stress concentration compared to cases where the HA coating alone is used $[45,46]$. Overall, the FGBM implant could satisfy important factors such as biocompatibility, bioactivity, strength, and stress continuity in the dental implant system. 


\section{Conclusion}

In summary, the effects of radial and axial FGBM dental implants on the bone stress distribution near the implantbone interface were successfully evaluated using FE analysis. The results of this research demonstrated that the optimal design of FGBM implant reduces stress concentration in the cortical and cancellous bones compared to the conventional titanium one. This finding indicates that FGBM dental implant benefits in reducing the stress concentration in the bone, and this would advance the early stability of dental implant system. Moreover, it was found that FGBM parameters can play a pivotal role in achieving an optimized biomechanical function of FGBM dental implant in terms of stress distribution through the hosting bone. In other words, they are useful parameters from a design point of view in a way that they can be tailored to specific biomechanical and clinical applications for controlling stresses.

Conflicts of interest: None declared.

Funding: This research received no specific grant from any funding agency in the public, commercial, or not-forprofit sectors.

\section{References}

[1] Demenko V, Linetskiy I, Nesvit K, Hubalkova H, Nesvit V, Shevchenko A. Comput. Methods Biomech. Biomed. Eng. 2014, 17, 443-449.

[2] Demenko V, Linetsky I, Nesvit V, Linetska L, Shevchenko A. Comput. Methods Biomech. Biomed. Eng. 2014, 17, 1751-1761.

[3] Hasan I, Bourauel C, Keilig L, Reimann S, Heinemann F. Comput. Methods Biomech. Biomed. Eng. 2011, 14, 1113-1116.

[4] Kong L, Zhao Y, Hu K, Li D, Zhou H, Wu Z, Liu B. Adv. Eng. Software 2009, 40, 474-478.

[5] Perez MA, Frutos JC, Bea JA, Doblare M. Comput. Methods Biomech. Biomed. Eng. 2012, 15, 263-273.

[6] Christenson EM, Anseth KS, van den Beucken JJJP, Chan CK, Ercan B, Jansen JA, Laurencin CT, Li WJ, Murugan R, Nair LS, Ramakrishna S, Tuan RS, Webster TJ, Mikos AG. J. Orthop. Res. 2007, 25, 11-22.

[7] An B, Wang R, Arola D, Zhang D. J. Mech. Behav. Biomed. Mater. 2012, 9, 63-72.

[8] Li WJ, Laurencin CT, Caterson EJ, Tuan RS, Ko FK. J. Biomed. Mater. Res. A 2002, 60, 613-621.

[9] Leong KF, Chua CK, Sudarmadji N, Yeong WY. J. Mech. Behav. Biomed. Mater. 2008, 1, 140-152.

[10] Askari E, Mehrali M, Metselaar IHSC, Kadri NA, Rahman MM. J. Mech. Behav. Biomed. Mater. 2012, 12, 144-150.

[11] Pompea W, Worch H, Epple M, Friess W, Gelinsky M, Greil P, Hempel U, Scharnweber D, Schulte K. Mater. Sci. Eng. A 2003, 362, 40-60.
[12] Virdee SS, Wang FC, Xu H, Jin ZM. Proc. IMechE Part H J. Eng. Med. 2003, 217, 191-198.

[13] Enab TA. Comput. Mater. Sci. 2012, 53, 94-100.

[14] Oshkour AA, Abu Osman NA, Yau YH, Tarlochan F, Wan Abas WAB. Proc. IMechE Part H J. Eng. Med. 2012, 227, 3-17.

[15] Hedia HS, Fouda N. Comput. Mater. Sci. 2014, 87, 83-87.

[16] Asgharzadeh Shirazi H, Ayatollahi MR. Int. J. Eng. Sci. 2014, 84, 62-78.

[17] Nayak AK. Int. J. Chem. Tech. Res. 2010, 2, 903-907.

[18] Cengiz B, Gokce Y, Yildiz N, Aktas Z, Calimli A. Colloids Surf. A 2008, 322, 29-33.

[19] Monmaturapoj N. J. Metals Mater. Miner. 2008, 18, 15-20.

[20] Bakan F, Laçin O, Sarac H. Powder Technol. 2012, 233, 295-302.

[21] Kumar GS, Thamizhavel A, Girija E. Mater. Lett. 2012, 76, 198-200.

[22] El Asri S, Laghzizil A, Saoiabi A, Alaoui A, El Abassi K, M’hamdi R, Coradin T. Colloids Surf. A 2009, 350, 73-78.

[23] Herliansyah M, Hamdi M, Ide-Ektessabi A, Wildan MW, Toque JA. MateR. Sci. Eng. C 2009, 29, 1674-1680.

[24] Asgharzadeh Shirazi H, Ayatollahi MR, Naimi-Jamal MR. J. Mater. Eng. Perf. 2015, 24, 3300-3306.

[25] Ramesh S, Aw K, Tolouei R, Amiriyan M, Tan CY, Hamdi M, Purbolaksono J, Hassan MA, Teng WD. Ceram. Int. 2013, 39, 111-119.

[26] Ayatollahi MR,Yazid Yahya M, Asgharzadeh Shirazi H, Abu Hassan S. Ceram. Int. 2015, 41, 10818-10827.

[27] Watari F, Yokoyama A, Saso F, Uo M, Kawasaki T. Compos. Part B Eng. 1997, 28, 5-11.

[28] Watari F, Yokoyama A, Saso F, Uo M, Matsuno H, Kawasaki T. J. JPN I Met. 1998, 62, 1095-10101.

[29] Watari F, Yokoyama A, Omori M, Hirai T, Kondo H, Uo M, Kawasaki T. Compos. Sci. Technol. 2004, 64, 893-908.

[30] Hedia H, Mahmoud NA. Bio-Med. Mater. Eng. 2004, 14, 133-143.

[31] Hedia H. J. Biomed. Mater. Res. Part B Appl. Biomater. 2005, 75, 74-80.

[32] Yang J, Xiang HJ. J. Biomech. 2007, 40, 2377-2385.

[33] Lin D, Li Q, Li W, Swain M. J. Biomed. Mater. Res. Part B Appl. Biomater. 2010, 92, 430-438.

[34] Eraslan A, Akis T. Acta Mech. 2006, 181, 43-63.

[35] Chi SH, Chung YL. Int. J. Solids Struct. 2006, 43, 3657-3674.

[36] Ichim IP, Schmidlin PR, Li Q, Kieser JA, Swain MV. Dent. Mater. 2007, 23, 1562-1569.

[37] Ichim IP, Kieser JA, Swain MV. Arch. Oral Biol. 2007, 52, 465-473.

[38] Kong L, Liu B, Li D, Song Y, Zhang A, Dang F, Qin X, Yang J. World J. Model Simu. 2006, 2, 134-140.

[39] Misch CE, Steigenga J, Barboza E, Misch-Dietsh F, Cianciola LJ, Kazor C. J. Peridontol. 2006, 77, 1340-1347.

[40] Ao J, Li T, Liu Y, Ding Y, Wu G, Hu K, Kong L. Comput. Biol. Med. 2010, 40, 681-686.

[41] Meric G, Erkmen E, Kurt A, Tunc Y, Eser A. J. Dent. Sci. 2011, 6, 25-32.

[42] Chang HS, Chen YC, Hsieh YD, Hsu ML. J. Dent. Sci. 2013, 8, 261-271.

[43] Chen LJ, He H, Li YM, Li T, Guo X-p, Wang R-f. T. Nonferr. Metal. Soc. 2011, 21, 1602-1610.

[44] Kayabaşı O, Yüzbasıoğlu E, Erzincanlı F. Adv. Eng. Software 2006, 37, 649-658.

[45] We M, Ruys A, Swain M, Milthorpe BK, Sorrell CC. J. Mater. Sci Mater. Med. 2005, 16, 101-106.

[46] Roy M, Bandyopadhyay A, Bose S. Surf. Coat Technol. 2011, 205, 2785-2792. 